\title{
A Study on the Efficiency Improvements of the Modified Design Propeller Pump Using Numerical Methods
}

\author{
Hee Jae Shin ${ }^{1}$,Sun Ho Go ${ }^{2}$, Lee Ku Kwac ${ }^{3}$,Hong Gun Kim ${ }^{4}$ \\ ${ }^{I}$ (Institute of Carbon Technology, JeonjuUniversity, South Korea ) \\ ${ }^{2}$ (Department of Mechanical Engineering, Jeonju University, South Korea) \\ ${ }_{3}^{3}$ (Department of Manufacturing and Design Engineering, JeonjuUniversity, South Korea) \\ ${ }^{4}$ (Institute of Carbon Technology, JeonjuUniversity, South Korea)
}

\begin{abstract}
Typically, in order to prevent the rainwater flooding of populated areas and limit the large water circulation in pumps for power plants, flooding of farmland, the propeller pumps used primarily introduced for drainage and sewage treatment are consist of large suction casing, impeller, discharge casing, and motor. When the main shaft and the impeller rotate and driven by an electric motor, the fluid sucked through the suction casing has the principle to be sent to the outside through the impeller and the guide vane. At this time most of the propeller pump that have T shape, severe vibration and vortex the drop of efficiency of the pump. Therefore, in this paper, we increased the mechanical efficiency through the change of the design and through the numerical methods for the impact and efficiency on the lift flow characteristics, the Reynolds number was derived and the feasibility of reducing turbulence and vibration was analyzed.
\end{abstract}

Keywords : computational fluid dynamics, propeller pump, shape design, total head

\section{Introduction}

Propeller pump is one of the representative fluid machines that coverts mechanical energy to pressure and kinetic energy of fluids by employing a rotating impeller which is being driven by external powertrain. Selected in the case where delivery capacity is extremely huge $\left(100 \sim 400 \mathrm{~m}^{3} / \mathrm{min}\right.$ on average) but total pump head is low (less than $20 \mathrm{~m}$ on average), the pump is appropriate for massive drainage way (rain water pump facility) or irrigated water supply facility. Blades of the impeller are large and wide and have the shape of a propeller. The lifting force generated by the rotation of the blades provides pressure and velocity to the fluids, when extreme swirling and vibration occur in the case of a conventional T-pipe. Therefore, this research paper is aimed at taking a numerical interpretation approach to ensure smooth water flow by changing the shape of the outlet and lowering swirling and vibration as such thereby improving overall efficiency $[1,2]$.

\subsection{Hydrodynamic theory of propeller pumps}

\section{Theoretical Background}

Menter'sk- $\omega$ SST turbulence model was applied for the numerical interpretation [3]. The drawback of Wilcox model is in general its sensitivity to free streamline. In the fluent, however, $\mathrm{k}-\omega$ model was adopted near the walls in an attempt to make up for such drawback whereas BSL (baseline model) and SST (Shear Stress Transport) models which both use k- $\varepsilon$ model were supported in the outer side [4].In this paper, $k-\omega$ SST model was applied to determine Eddy-Viscosity of the turbulence while conditions as specified in Equation (1) were assumed to prevent excessive prediction.

$$
v_{T}=\frac{0.31 k}{\max \left(0.31 w ; \Omega F_{2}\right)}(1)
$$

Where :

$\Omega=$ Absoulute Value of Vorticity

$F_{2}=\tanh \left(\left(\arg _{2}\right)^{2}\right), \arg _{2}=\max$

\subsection{Computing performance efficiency of the propeller pump}

The efficiency of pump is expressed with the ratio of hydraulic power per axial power, whereas the hydraulic power is expressed with mass flow $\left(\mathrm{Q}_{\mathrm{m}}\right)$, water density $(\rho)$, gravity $(\mathrm{g})$ and total pump head $\left(\mathrm{h}_{\mathrm{t}}\right)$ as shown in Equation (2). It is also expressed with such parameters as entrance voltage $\left(\mathrm{P}_{\mathrm{t}_{\mathrm{i}}}\right)$, exit voltage $\left(\left(\mathrm{P}_{\mathrm{t}_{\mathrm{o}}}\right)\right.$, water density and gravity as shown in Equation (4). The axial power is expressed with the torque of the rotation area of the impeller as well as with the number of rotations of impeller while the unit of rotation is rad/s here.

$$
\eta_{p}=\frac{P_{\text {flow }}}{P_{\text {shaft }}}
$$




$$
\begin{aligned}
& P_{\text {flow }}=Q_{m} \times \rho \times h_{t} \\
& P_{\text {shaft }}=\text { Torque } \times \mathrm{rps} \\
& h_{t}=\frac{P_{t_{i}}-P_{t_{o}}}{\rho g}
\end{aligned}
$$

\section{Modeling and Analysis Conditions}

\subsection{Modeling of propeller pump}

The pump used in this paper is the one that is used for cooling systems which operate using river or sewage water as working fluid. The pump is composed of the inlet that is used as a passage for sucking in river water which is working fluid, the impeller which imparts energy to the fluids, casing assembly that converts screw motion of the fluid deviating from the impeller to axial motion and the outlet which turns dynamic pressure of the fluid into static pressure partially. Table 1 shows the design specifications of the pump that was actually used [5].

Table 1 Pump specifications

\begin{tabular}{|l|l|l|l|l|}
\hline Discharge Rate & Total Head & Pump Diameter & Number of rotation & Motor output \\
\hline $300 \mathrm{~m}^{3} / \mathrm{min}$ & $4.3 \mathrm{~m}$ & $1500 \mathrm{~mm}$ & $290 \mathrm{rpm}$ & $300 \mathrm{~kW}$ \\
\hline
\end{tabular}

Each part of the pump was turned to imaged and screws or gaps that do not exert direct influence on the interpretation were simplified partially to study the flow characteristics of the pump. Fig. 1 shows existing and newly designed modeling results whereas Fig. 2 shows respective flow field for each model.

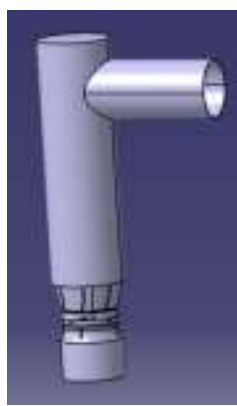

(a) Existing model

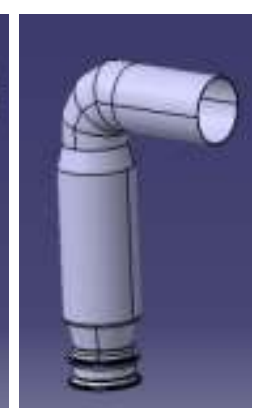

(b) Improve model(a) Existing model

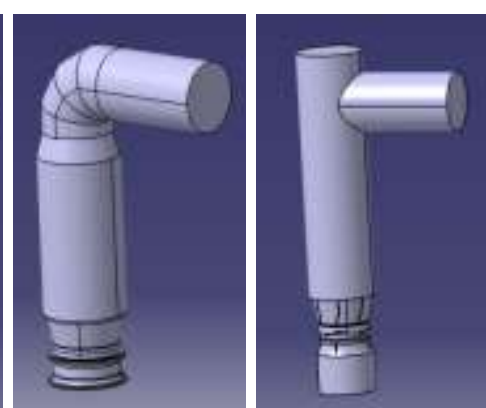

(b) Improve model

Figure 1.3 D modelingFigure 2.Flow area of 3D modeling

As the overall image represents the flow assembly, it was designated as fluid while the pump was divided into four parts including inlet through which river water is being sucked in, impeller which imparts energy to the fluids, casing which converts screw motion of the fluids into axial motion, and outlet which turns dynamic pressure of the fluids into static pressure partially. And in an attempt to secure pressure in the inlet and outlet and stability of the flow field, the flow fields were extended by $2.5 \mathrm{~m}$ in the outlet and $1 \mathrm{~m}$ in the inlet, respectively.
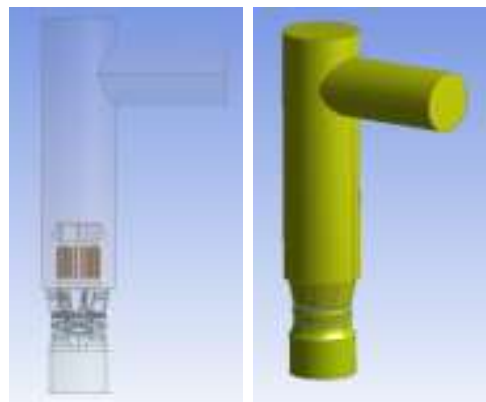

Figure 3. Flow modeling of existing model
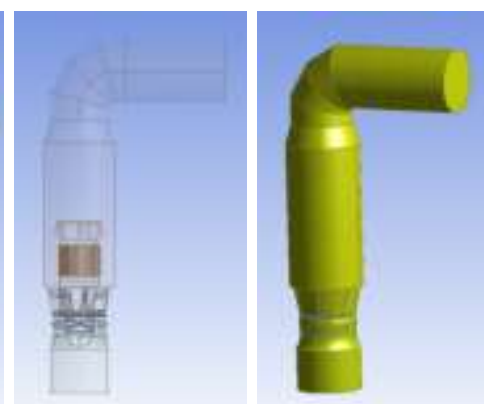

Figure 4. Flow modeling of improved model

\subsection{Mesh modeling and boundary conditions}

In this paper, the flow fields were formed by eliminating unnecessary elements to allow formation of high quality grid in a model with complicated shapes, while lots of grids were concentrated in the impeller and 
casing domain in consideration of the interpretation accuracy. In the case of such studies that require modification each time depending on the model that is to be interpreted and aim to analyze the impact of a few variables, the reliability in the data comparison can be ensure only when multiple grids with almost identical quality level should be created. Unstructured tetrahedral mesh was used mainly for grids while relatively smaller number of grids was placed in the inlet and outlet as compared to the impeller and casing assembly where flow speed and pressure are forecasted to fluctuate significantly. In this interpretation, the number of grid applied for the overall flow interpretation model was about $4.5 \mathrm{M}$ in consideration of density and error rate in the numerical interpretation of the grids [6].

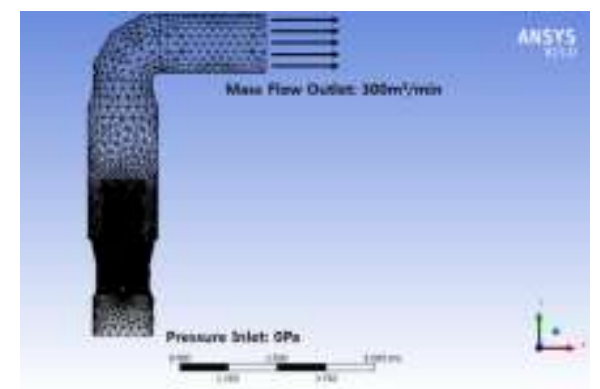

Figure 5. Boundary conditions (inlet, outlet)

The working fluid used in the interpretation was in the liquid state of water with its density being set at $998.2 \mathrm{~kg} / \mathrm{m}^{3}$ and viscosity at $0.001003 \mathrm{~kg} / \mathrm{m}$-s. And no slip condition was applied along entire walls whereas the impeller domain with the exception of inlet, casing and outlet domains was set as rotating domain. And the flow field was rotated at the speed of 290rpm, which is the default specification of the pump. And inlet space was created in the take whereas the pressure in the inlet surface was set at $0 \mathrm{~Pa}$.

And five different flows including $300 \mathrm{~m}^{3} / \mathrm{min}$, which is the design specification marked on the pump specification, as well as $315 \mathrm{~m}^{3} / \mathrm{min}, 266.11 \mathrm{~m}^{3} / \mathrm{min}, 235 \mathrm{~m}^{3} / \mathrm{min}$ and $200 \mathrm{~m}^{3} / \mathrm{min}$ were set as the fluid discharge conditions near the boundary interface of the pump outlet, thereby enabling verification of the changing pump performance depending on the change in the flow. For this interpretation, ANSYS Fluent 15.0V, which is commercial flow interpretation software, was used while thek- $\omega$ SST(Shear Stress Transport) model which allows relatively accurate prediction of the impact stemming from the secondary flow that is generated by flow separation among many applicable turbulence models as well as the impact from boundary interface in the walls was used. Calculation was performed by assuming a flow condition at normal state while remaining error amounting to $3 \times 10^{-4}$ was confirmed while checking convergent tendency of the remaining error [7].

\subsection{Analysis of velocity distribution}

\section{Analysis Results}

The speed distribution of the flow field of the pump when the design flow was $300 \mathrm{~m}^{3} / \mathrm{min}$ was shown in Fig. 6 The speed distribution pattern of the existing model and that of the model with changed design specification were completely different: the model with changed design demonstrated a rather smooth flow stream into the outlet but the actual speeds of flow were almost identical according to the interpretation results.

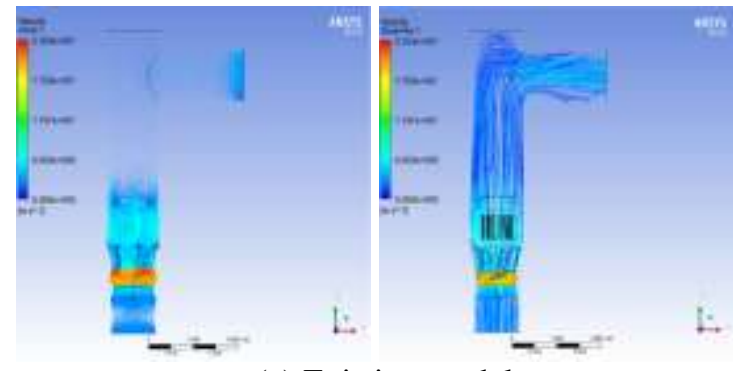

(a) Existing model

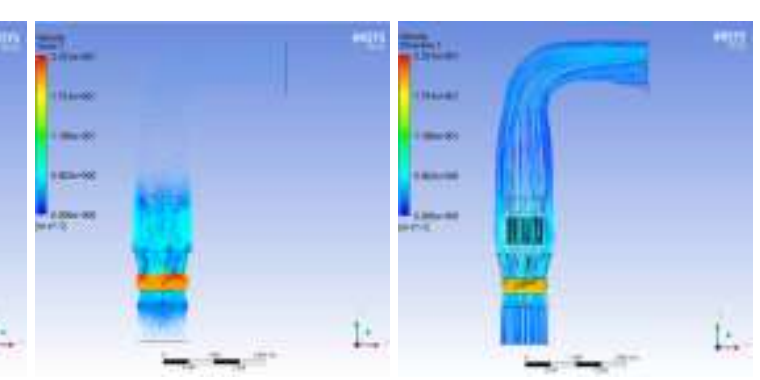

(b) Improved model

Figure 6, Distribution of flow velocity

\subsection{Analysis of total head, torque and efficiency}

Numerical interpretation was performed for five different delivery flows from $200 \mathrm{~m}^{3} / \mathrm{min}$ to $315 \mathrm{~m}^{3} / \mathrm{min}$ including the design flow in an attempt to maximize efficiency and derive an optimized discharge flow that satisfies the minimum total pump head height $(4 \mathrm{~m})$ based on the specifications. As it is confirmed in the performance graphs and interpretation results in Table 2, Table3, Fig. 7 and Fig. 8, the total pump head at 
the design flow of $300 \mathrm{~m}^{3} / \mathrm{min}$ was $4.5 \mathrm{~m}$ in the case of the existing model and $4.42 \mathrm{~m}$ in the model with design change but its efficiency was $76.19 \%$ in the existing model and $78.88 \%$ in the model with design change, thereby confirming about $2.69 \%$ improvement.

Table 2 Performance evaluation of existing model

\begin{tabular}{|l|l|l|l|l|}
\hline Discharge rate & Difference of total pressure & Total head & Torque & Efficiency \\
\hline $200 \mathrm{~m}^{3} / \mathrm{min}$ & $63,943.99 \mathrm{~Pa}$ & $6.53 \mathrm{~m}$ & $12,967.25 \mathrm{~N} . \mathrm{m}$ & $54.12 \%$ \\
\hline $235 \mathrm{~m}^{3} / \mathrm{min}$ & $58,264.43 \mathrm{~Pa}$ & $5.95 \mathrm{~m}$ & $11,453.01 \mathrm{~N} . \mathrm{m}$ & $65.611 \%$ \\
\hline $266.11 \mathrm{~m}^{3} / \mathrm{min}$ & $51,311.87 \mathrm{~Pa}$ & $5.24 \mathrm{~m}$ & $10,947.87 \mathrm{~N} . \mathrm{m}$ & $68.45 \%$ \\
\hline $300 \mathrm{~m}^{3} / \mathrm{min}$ & $44,065.53 \mathrm{~Pa}$ & $4.5 \mathrm{~m}$ & $9,522.68 \mathrm{~N} . \mathrm{m}$ & $76.45 \%$ \\
\hline $315 \mathrm{~m}^{3} / \mathrm{min}$ & $37,112.97 \mathrm{~Pa}$ & $3.79 \mathrm{~m}$ & $8,897.99 \mathrm{v}$ & $72.11 \%$ \\
\hline
\end{tabular}

Table 3 Performance evaluation of improved model

\begin{tabular}{|l|l|l|l|l|}
\hline Discharge rate & Difference of total pressure & Total head & Torque & Efficiency \\
\hline $200 \mathrm{~m}^{3} / \mathrm{min}$ & $64,727.38 \mathrm{~Pa}$ & $6.61 \mathrm{~m}$ & $12,446.66 \mathrm{~N} . \mathrm{m}$ & $57.08 \%$ \\
\hline $235 \mathrm{~m}^{3} / \mathrm{min}$ & $58,166.51 \mathrm{~Pa}$ & $5.94 \mathrm{~m}$ & $10,922.44 \mathrm{~N} . \mathrm{m}$ & $68.68 \%$ \\
\hline $266.11 \mathrm{~m}^{3} / \mathrm{min}$ & $50,920.18 \mathrm{~Pa}$ & $5.2 \mathrm{~m}$ & $10,589.37 \mathrm{~N} . \mathrm{m}$ & $70.23 \%$ \\
\hline $300 \mathrm{~m}^{3} / \mathrm{min}$ & $43,282.15 \mathrm{~Pa}$ & $4.42 \mathrm{~m}$ & $9,034.53 \mathrm{~N} . \mathrm{m}$ & $78.88 \%$ \\
\hline $315 \mathrm{~m}^{3} / \mathrm{min}$ & $36,721.28 \mathrm{~Pa}$ & $3.75 \mathrm{~m}$ & $8,562.98 \mathrm{~N} . \mathrm{m}$ & $74.14 \%$ \\
\hline
\end{tabular}

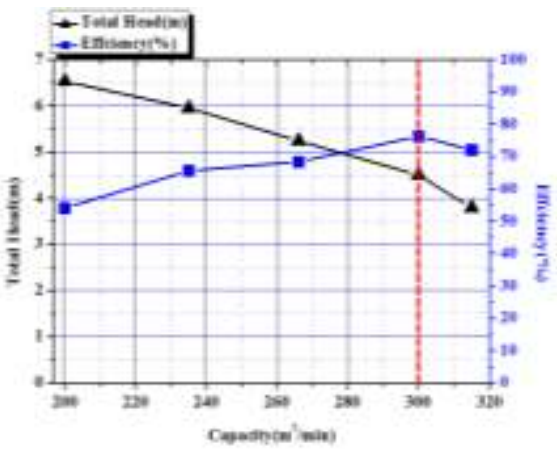

Figure 7. Total head Efficiency of existing model

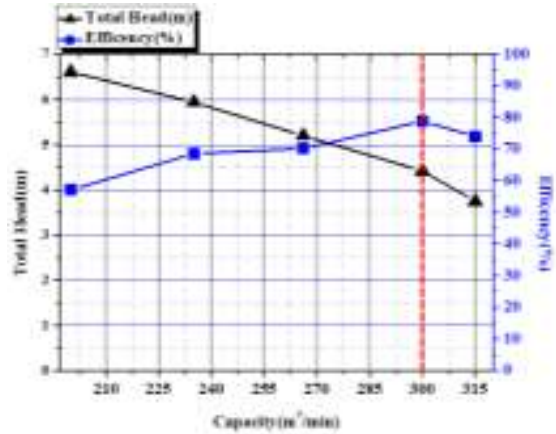

Figure 8. Total head Efficiency of improve model

As demonstrated in Fig.9, a narrower torque distribution was confirmed in the model with design change than in the existing model thereby supporting the conclusion that it contributed to the efficiency improvement. The total pump head was observed higher before and after the design change at the following discharge flows $266.11 \mathrm{~m}^{3} / \mathrm{min}, 235 \mathrm{~m}^{3} / \mathrm{min}, 200 \mathrm{~m}^{3} / \mathrm{min}$ but its efficiency was less than $70 \%$ level. In the case of discharge flow of $315 \mathrm{~m}^{3} / \mathrm{min}$, the efficiency was scaled down to $74.14 \%$ while its total pump head was calculated as $3.75 \mathrm{~m}$ thereby failing to meet the design specification. In conclusion, it was confirmed that the discharge flow of $300 \mathrm{~m}^{3} / \mathrm{min}$ is the optimized flow.

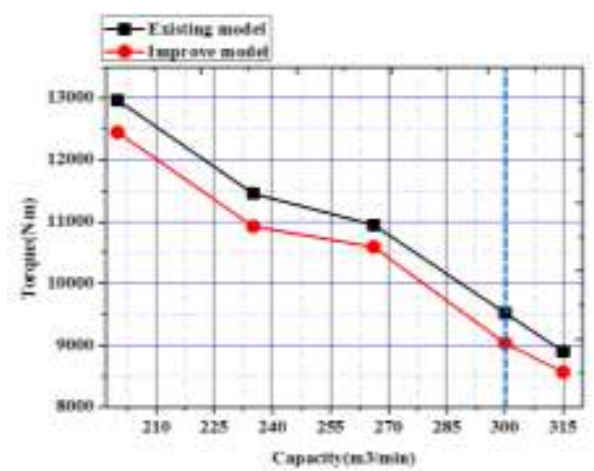

Figure 9. Comparison of torque according to flow rate

\subsection{Analysis of reynolds number and turbulent kinetic energy}

In an attempt to identify the turbulence minimization performance using the optimized model, Reynolds' number and turbulence motion energy were compared, respectively. As shown in Fig. 10 and Fig. 11, Reynolds' number of the existing model was 554,984.9 whereas that of the model with design change was $525,320.6$, thereby confirming about $5.35 \%$ reduction. 

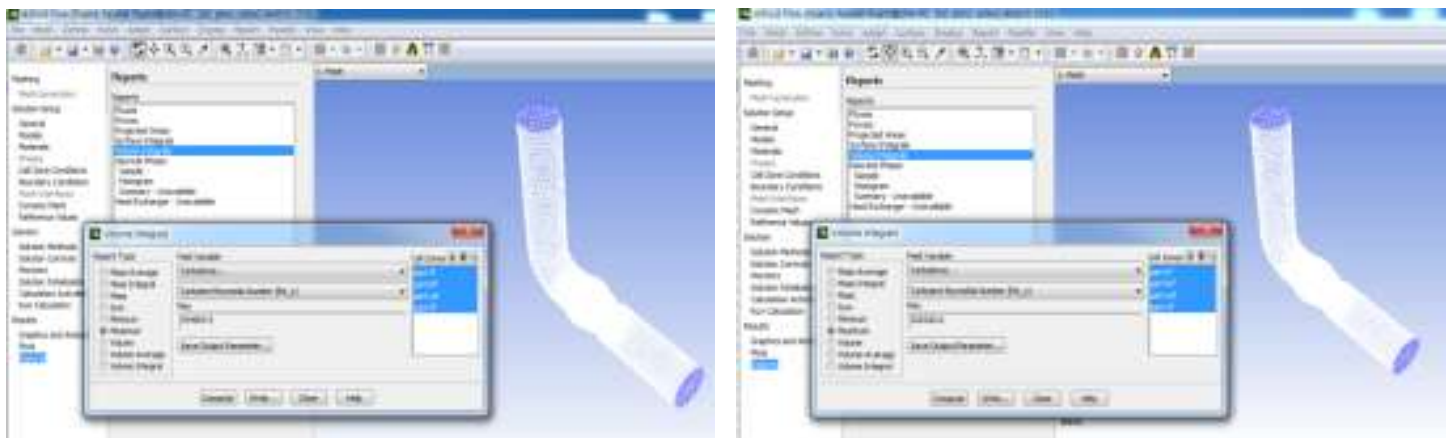

Figure 10.Existing model of the Reynolds number Figure 11. Improved model of the Reynolds number

In the case of turbulence energy, an analysis depending on the location of the inlet and outlet was performed. The result shows that the kinetic energy measured at locations inside the pump some $5.5 \mathrm{~m}, 6.5 \mathrm{~m}$ and $7.5 \mathrm{~m}$ from the inlet was lower than those measured in the newly designed pump with the results shown in Fig. 12 through Fig. 14.

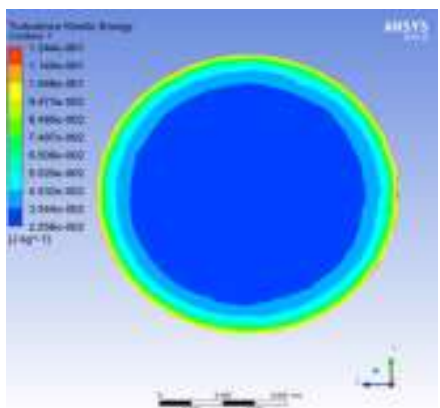

(a) Existing model

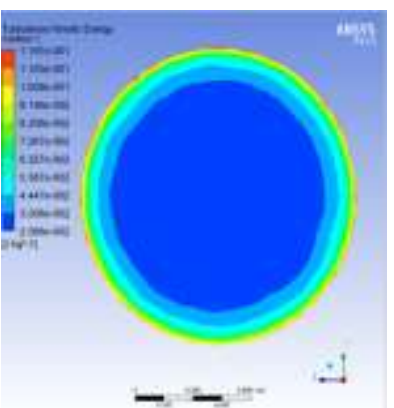

(b) Improved model

Figure 12. Turbulent kinetic energy of the location away from the inlet to $5.5 \mathrm{~m}$

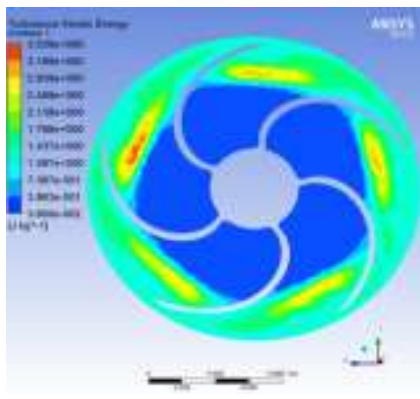

(a) Existing model

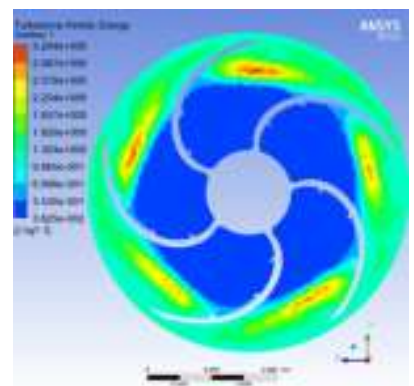

(b) Improved model

Figure 13. Turbulent kinetic energy of the location away from the inlet to $6.5 \mathrm{~m}$

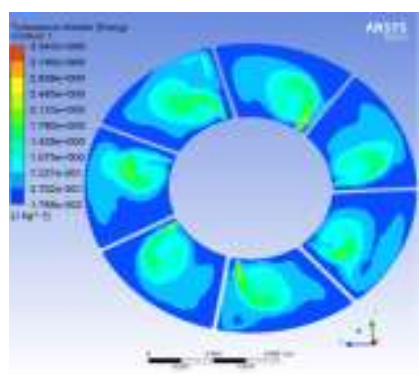

(a) Existing model

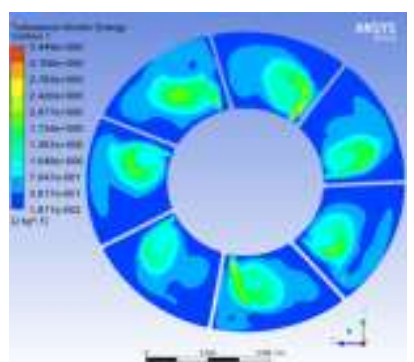

(b) Improved model

Figure 14.Turbulent kinetic energy of the location away from the inlet to $7.5 \mathrm{~m}$

In the case of outlet, the kinetic energy measured at locations inside the pump some $0 \mathrm{~m}, 1 \mathrm{~m}$ and $2 \mathrm{~m}$ from the outlet was lower than those of the newly designed pump with the results shown in in Fig. 15 through Fig. 17 and the data analysis table depending on the location of inlet and outlet shown in Table 4. 


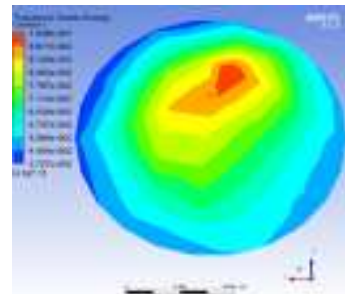

(a) Existing model

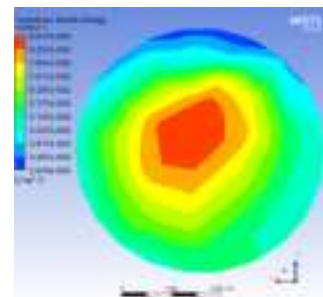

(b) Improved model

Figure 15. Turbulent kinetic energy of the location away from the outlet to $0 \mathrm{~m}$

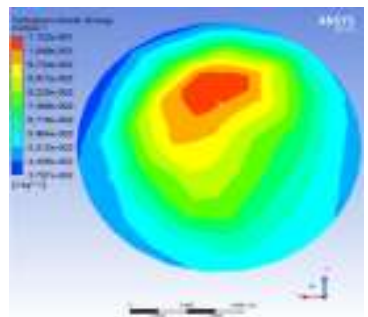

(a) Existing model

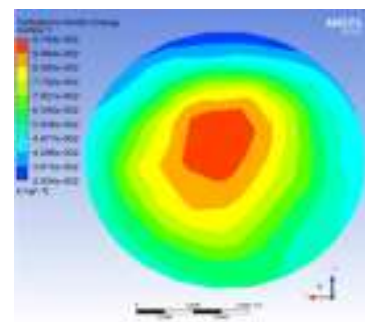

(b) Improved model

Figure 16. Turbulent kinetic energy of the location away from the outlet to $1 \mathrm{~m}$

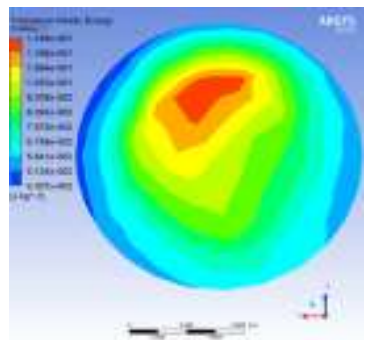

(a) Existing model

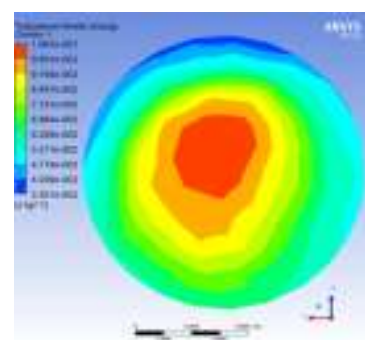

(b) Improved model

Figure 17. Turbulent kinetic energy of the location away from the outlet to $1 \mathrm{~m}$

Table 4 Comparison of turbulent kinetic energy

\begin{tabular}{|l|l|l|l|l|l|}
\hline Inlet & Outlet \\
\hline Displacement & Existing model & Improved model & Displacement & Existing model & Improved model \\
\hline $5.5 \mathrm{~m}$ & $12.44 \mathrm{~J} / \mathrm{kg}$ & $11.97 \mathrm{~J} / \mathrm{kg}$ & $0 \mathrm{~m}$ & $0.105 \mathrm{~J} / \mathrm{kg}$ & $0.089 \mathrm{~J} / \mathrm{kg}$ \\
\hline $6.5 \mathrm{~m}$ & $3.54 \mathrm{~J} / \mathrm{kg}$ & $3.20 \mathrm{~J} / \mathrm{kg}$ & $1 \mathrm{~m}$ & $0.123 \mathrm{~J} / \mathrm{kg}$ & $0.097 \mathrm{~J} / \mathrm{kg}$ \\
\hline $7.5 \mathrm{~m}$ & $3.54 \mathrm{~J} / \mathrm{kg}$ & $3.45 \mathrm{~J} / \mathrm{kg}$ & $2 \mathrm{~m}$ & $0.125 \mathrm{~J} / \mathrm{kg}$ & $0.107 \mathrm{~J} / \mathrm{kg}$ \\
\hline
\end{tabular}

\subsection{Analysis of shear distribution of propeller pump}

The coefficient of shear distribution of the newly designed propeller pump was checked to identify its vibration characteristics. Whereas the maximum coefficient of shear distribution of the existing pump was $1,164 \mathrm{~Pa}$, that of the newly designed pump was 1,215 thereby confirming some $4.2 \%$ reduction, with the result shown in Fig. 18.based on the aforementioned results, it is judged that the newly designed pump would be able to reduce vibration as discontinuous swirling vortex stemming from the collision of shearing force is minimized, and minimize vibration by reducing generation of pressure waves or pressure pulse.

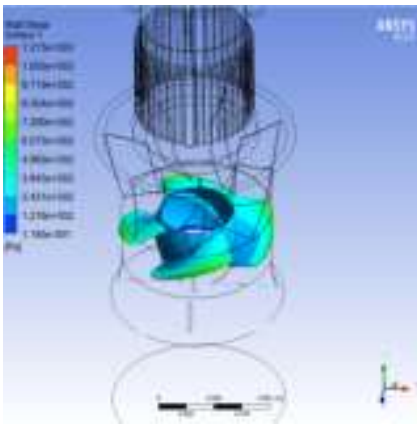

(a) Existing model

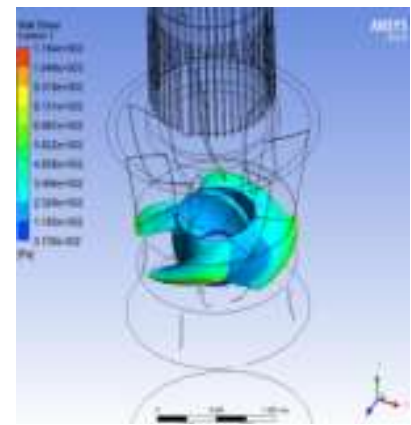

(b) Improved model

Figure 17. Value of wall shear 


\section{Conclusion}

In this study, a pump design was conducted in an attempt to reduce vibration and noise through efficiency maximization and vibration wave minimization of the existing propeller pump. Towards this end, a flow analysis was performed followed by an analysis of the pump efficiency, Reynolds' number and turbulence kinetic energy.The total pressure difference, total pump head and torque were each calculated to identify the performance of the propeller pump thereby analyzing changing efficiency depending on the flow amount. After confirming some $2.69 \%$ efficiency improvement at discharge flow of $300 \mathrm{~m}^{3} / \mathrm{min}$, a performance optimization was implemented.The maximum Reynolds' number was calculated to check extent of turbulence reduction as compared to the existing pumps with some 5.35\% reduction as compared to that of the existing model confirmed.Turbulence kinetic energy at locations apart from the inlet and outlet were measured to analyze the strength of the turbulence. Numbers that are significantly lower than the existing results were calculated by analyzing the distribution of turbulence kinetic energy at locations some 5 to $7 \mathrm{~m}$ from the inlet and 0 to $2 \mathrm{~m}$ from the outlet.Vortex stream is generated when the fluid flows to a resisting body and pressure of the flow particles increases from the fluid pressure level to the static pressure level. And the interface boundary is formed near the location where the fluid pressure is highest at the resisting body and the stream of the shearing force layer adjacent to the surface of the resisting body is markedly slower than the shearing force layer at the outermost side facing the flow that is uninhibited by the stream. For this reason, the collision of the shearing force generates non-continuous swirling vortex while turning as well as high and low pressure waves or pulse waves. Therefore, the higher the shearing force is, the larger the vibration and noise level becomes. To analyze such phenomenon, the shearing force distributions along the walls surrounding the impeller and inlet axle stream pump were compared. It was confirmed that the shearing force was reduced by $4.15 \%$ than that of the existing model thereby confirming turbulence reduction as well as vibration and noise reduction.

\section{Acknowledgements}

This research was supported by basic science research program through the National Research Foundation of Korea(NRF) funded by the ministry of education, science and technology (No.2013R1A1A2061581),(No.2013R1A1A2062899) and financially supported by the National Foundation of Korea(NRF) funded by the Korea Government(MISP) (No.2014R1A2A1A11054594).

\section{References}

[1] H. S. Lee, H. S. Kang and D. J. Jung, H. H. Yun, Y. M. Park and G. J. Oh, Performance Analysis of the Submerged Axial-Flow Pump, The Korean Society of Mechanical Engineers Annual Autumn Conference, 2014, 393-398.

[2] W. B. Jung and S. Kim and G. Y. Lee and J. H. Kim and Y. S. Choi and J. Y. Yun, Design Optimization of Inlet Meridional Shape for Mixed-flow Pump Impeller Using RSM, Korean Society For Fluid Machinery, 2015, 97-98.

[3] J. H. Kim and Y. D. Choi, Internal Flow Analysis on a Mixed-Flow pump, The Korean Society of Mechanical Engineers Annual Autumn Conference, 2014, 47-48.

[4] J. Y. Kim and G. R. Lee and S. I. Jung, Numerical Study on the Internal Leakage Flow for Mixed-flow Pump, Korean Society For Fluid Machinery, 2015, 222-223.

[5] H. R. Yoo and W. G. Park, Numerical Flow Analysis of Propeller Type Pump, Korean Society For Fluid Machinery, 9(6), 2016, 2934.

[6] H. D. Lee and Y. G. Seo, Study on the Effect of the Impeller Diameter on the Performance of a Mixed-flow Pump, Journal of KSFM, 15(4), 2012, 61-66.

[7] J. W. Lee and Y. D. Choi and Y. H. Lee, C. H. Yun, J. M. Park, Internal Flow Analysis on a Mixed Flow Pump for Developing Marine Mineral Resources, Korean Society For Fluid Machinery, 13(5), 2010, 11-16. 